Supporting information

\title{
Gel-Modulated Growth of High-quality Zeolite Membranes
}

Ji Jiang ${ }^{1}$, Qiaobei Dong ${ }^{1}$, Fanglei Zhou ${ }^{1}$, Weiwei $\mathrm{Xu}^{1}$, Shiguang $\mathrm{Li}^{2}$, Miao $\mathrm{Yu}^{1 *}$

1 Department of Chemical \& Biological Engineering, Rensselaer Polytechnic

Institute, Troy, New York 12180, USA

2 Gas Technology Institute, 1700 S Mount Prospect Road, Des Plaines, IL 60018,

$U S A$

Corresponding author: Miao Yu (yum5@rpi.edu) 
Table S1. Supernatant gel Al/Si/P molar ratio (after quenching step) after different membrane "skeleton" construction time.

\begin{tabular}{cccccc}
\hline $\begin{array}{c}\text { Synthesis } \\
\text { time }(\mathrm{h})\end{array}$ & 1 & 2 & 3 & 4 & 5 \\
\hline $\begin{array}{c}\mathrm{Al} / \mathrm{Si} / \mathrm{P} \\
\text { ratio }\end{array}$ & $1: 0.45: 1.62$ & $1: 0.44: 1.65$ & $1: 0.41: 1.66$ & $1: 0.40: 1.67$ & $1: 0.39: 1.68$ \\
\hline
\end{tabular}


Table S2. Synthesis time in crystal merging step on the separation performance of SAPO-34 membranes

\begin{tabular}{ccc}
\hline $\begin{array}{c}\text { Crystal merging } \\
\text { growth time }(\mathrm{h})\end{array}$ & $\mathrm{H}_{2}$ permeance $\left(\times 10^{-7} \mathrm{~mol} \cdot \mathrm{m}^{-2} \cdot \mathrm{s}^{-1} \cdot \mathrm{Pa}^{-1}\right)$ & $\begin{array}{c}\mathrm{H}_{2} / \mathrm{N}_{2} \\
\text { selectivity }\end{array}$ \\
\hline 4 & $25.21 \pm 2.12$ & $3.1 \pm 1.2$ \\
8 & $9.25 \pm 1.17$ & $7.6 \pm 2.8$ \\
12 & $7.57 \pm 0.23$ & $22.1 \pm 2.2$ \\
16 & $7.53 \pm 0.21$ & $22.3 \pm 2.7$ \\
20 & $7.54 \pm 0.17$ & $22.9 \pm 2.8$ \\
24 & $7.56 \pm 0.25$ & $23.1 \pm 2.7$ \\
\hline
\end{tabular}

Note: $\mathrm{H}_{2} / \mathrm{N}_{2}$ separation was performed at room temperature and pressure drop of 5 bar. Membrane "skeleton" construction step synthesis condition: $220^{\circ} \mathrm{C}$ for $3 \mathrm{~h}$. 
Table S3. Single-gas and mixture gas $\left(\mathrm{H}_{2} / \mathrm{N}_{2}\right)$ permeation results of SAPO-34 membranes prepared in different batches

\begin{tabular}{|c|c|c|c|c|}
\hline \multirow{2}{*}{$\begin{array}{c}\text { Membrane } \\
\text { batch }\end{array}$} & \multicolumn{2}{|c|}{$\mathrm{H}_{2}$ permeance $\left(\times 10^{-7} \mathrm{~mol} \cdot \mathrm{m}^{-2} \cdot \mathrm{s}^{-1} \cdot \mathrm{Pa}^{-1}\right)$} & \multirow{2}{*}{$\begin{array}{c}\mathrm{H}_{2} / \mathrm{N}_{2} \text { mixture } \\
\text { selectivity }\end{array}$} & \multirow{2}{*}{$\begin{array}{l}\mathrm{H}_{2} / \mathrm{N}_{2} \text { ideal } \\
\text { selectivity }\end{array}$} \\
\hline & Mixture & Single gas & & \\
\hline 1 & $7.52 \pm 0.32$ & $8.29 \pm 0.42$ & $23.2 \pm 2.9$ & $26.2 \pm 2.9$ \\
\hline 2 & $7.43 \pm 0.28$ & $8.15 \pm 0.29$ & $24.2 \pm 1.8$ & $27.4 \pm 1.8$ \\
\hline 3 & $7.62 \pm 0.45$ & $8.39 \pm 0.56$ & $22.7 \pm 3.5$ & $25.7 \pm 3.9$ \\
\hline 4 & $7.56 \pm 0.25$ & $8.25 \pm 0.30$ & $23.1 \pm 2.7$ & $26.8 \pm 2.1$ \\
\hline
\end{tabular}

Note: Single-gas and mixture gas $\left(\mathrm{H}_{2} / \mathrm{N}_{2}\right)$ separation were performed at room temperature and pressure drop of 5 bar. Membrane "skeleton" construction step synthesis condition: $220{ }^{\circ} \mathrm{C}$ for 3 h. 4-6 membranes were prepared in one batch. 

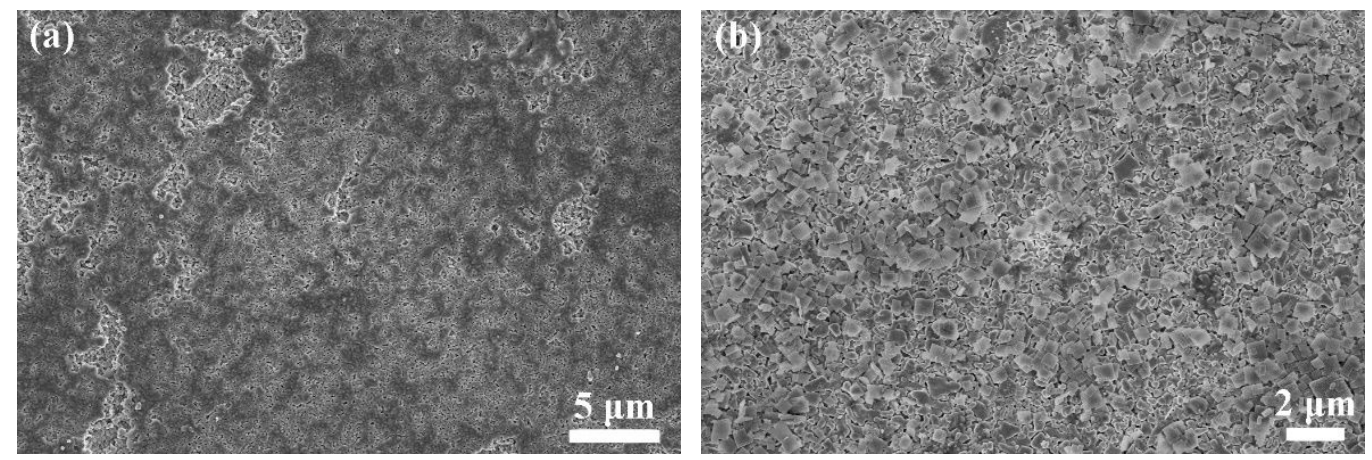

Figure S1. Surface SEM images of (a) $\mathrm{Al}_{2} \mathrm{O}_{3}$ hollow fiber support and (b) seeded support. 

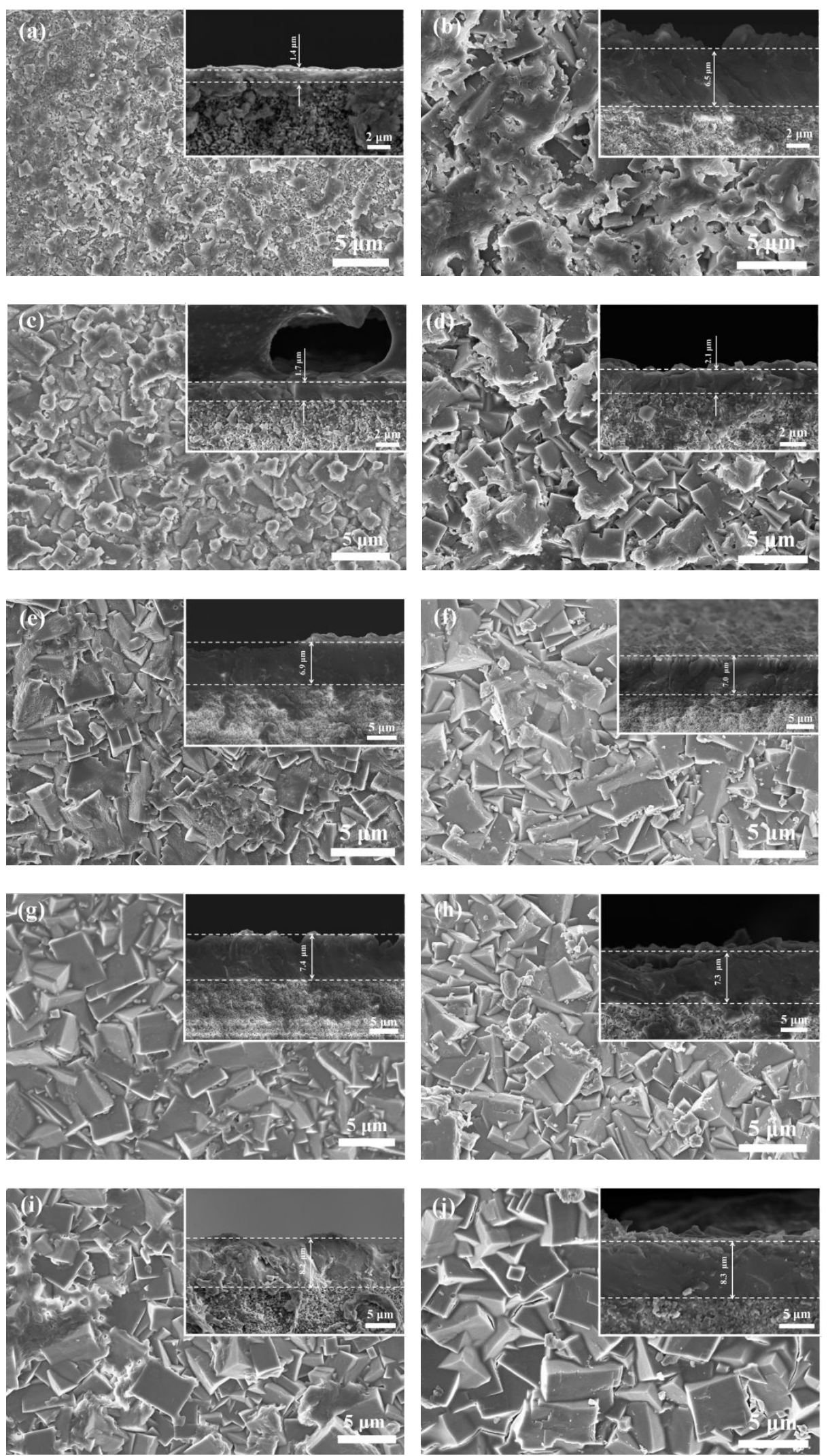

Figure S2. SEM images for SAPO-34 membranes prepared in membrane "skeleton" construction step for (a) $1 \mathrm{~h}$, (c) $2 \mathrm{~h}$, (e) $4 \mathrm{~h}$, (g) $5 \mathrm{~h}$, (i) $6 \mathrm{~h}$ and after crystal merging step for $24 \mathrm{~h}(\mathrm{~b}, \mathrm{~d}, \mathrm{f}, \mathrm{h}$ and $\mathrm{j})$ 


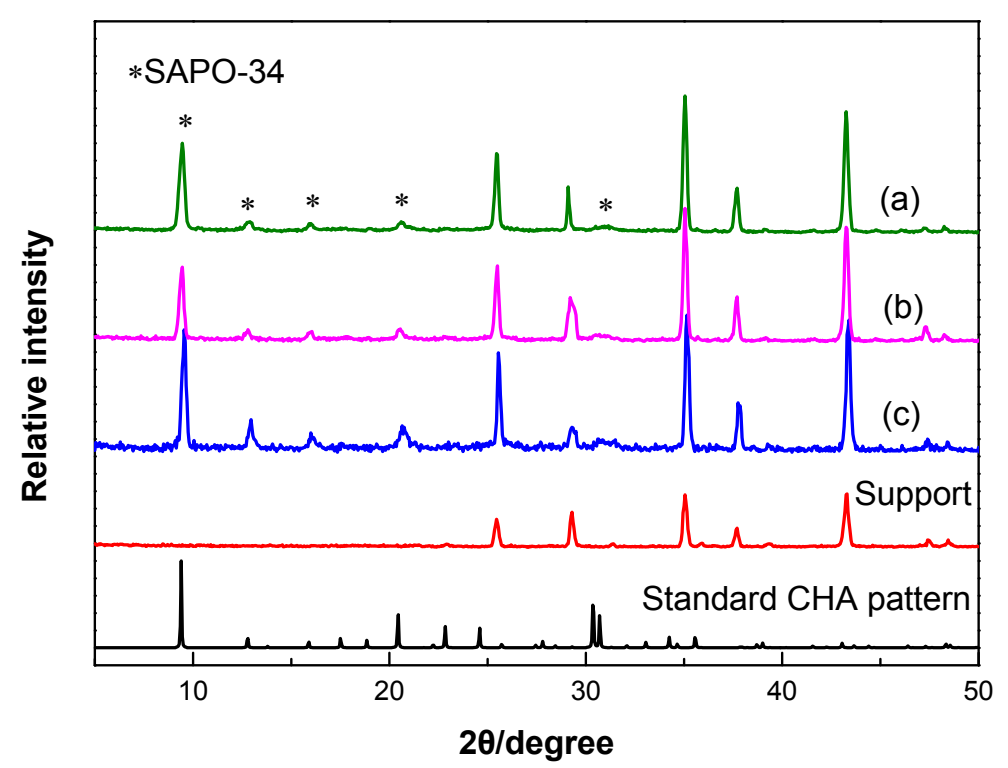

Figure S3. XRD results for SAPO-34 membranes prepared by gel modulated growth method with crystal merging time of (a) $12 \mathrm{~h}$, (b) $20 \mathrm{~h}$, and (c) $24 \mathrm{~h}$. Synthesis time in membrane "skeleton" construction step was controlled at $3 \mathrm{~h}$. 